\title{
Clinical usefulness of mepolizumab in severe eosinophilic asthma
}

\author{
This article was published in the following Dove Press journal: \\ Therapeutics and Clinical Risk Management \\ 8 June 2016 \\ Number of times this article has been viewed
}

\section{Francesco Menzella \\ Mirco Lusuardi \\ Gloria Montanari \\ Carla Galeone \\ Nicola Facciolongo \\ Luigi Zucchi}

Department of Cardio-ThoracicVascular and Intensive Care Medicine, Pneumology Unit, IRCCS Arcispedale Santa Maria Nuova, Reggio Emilia, Italy
Correspondence: Francesco Menzella Department of Cardio-Thoracic-Vascular and Intensive Care Medicine, Pneumology Unit, IRCCS - Arcispedale Santa

Maria Nuova, Viale Risorgimento 56, 42123 Reggio Emilia, Italy

Tel +390522 296073

Fax +390522 296 I82

Email menzella.francesco@asmn.re.it
Abstract: Asthma is a chronic inflammatory disorder of the airways with variable clinical severity from very mild and occasional symptoms to recurrent critical exacerbations, at risk of fatal or near-fatal outcome, in a small percentage of patients. Within the different inflammatory cascades involved in asthma, eosinophils play a central role in the pathogenesis and largely influence disease severity. Interleukin-5 (IL-5) is the main cytokine controlling eosinophil activity and proliferation at the site of inflammation. Mepolizumab was the first biological humanized anti-IL-5 monoclonal antibody tested in randomized clinical trials on eosinophilic asthma and other eosinophilic diseases. On the basis of several positive clinical efficacy data, it has recently been approved by the US Food and Drug Administration for the treatment of severe eosinophilic asthma. Unfortunately, high costs are at present a critical issue. Future studies will probably help in the correct selection of a potential "responder phenotype", allowing the prescription of this promising therapy to appropriate patients and best define cost-effectiveness issues.

Keywords: asthma, mepolizumab, phenotype, IL-5, effectiveness, biomarkers

\section{Introduction}

Asthma is a chronic inflammatory disorder of the airways with a large range of clinical severity from very mild and occasional symptoms to critical exacerbations in a small percentage of patients, at risk of fatal or near-fatal outcome. Severe asthma is often associated with persisting daily symptoms, poor quality of life, and high risk of exacerbations despite regular treatment with high doses of inhaled steroids and best usual care.

The prevalence of a severe form of the disorder affects less than $10 \%$ of patients but is associated with significant morbidity and mortality and a large proportion of the health care costs for the disease..$^{1-3}$

Within the different inflammatory pathways involved in asthma, eosinophils play a central role in the pathogenesis and largely influence disease severity. For more than a decade, research has tried to identify specific cytokines or other biological markers to guide the pharmacological therapy of subjects with severe asthma. Indeed, these patients require complex treatment, including in 30\%-40\% of cases who regularly use oral glucocorticoids to control symptoms and exacerbations, potentially inducing ${ }^{4-6}$ serious and frequently irreversible side effects. ${ }^{7}$ Safe glucocorticoid-sparing treatments would actually imply a large benefit in managing severe asthma. ${ }^{8}$

Asthma is not a unique disease but a syndrome with a heterogeneity of phenotypes or endotypes, with distinct cellular and biomolecular mechanisms. The prevalence of eosinophilic inflammation identifies one phenotype of asthma sustained by the biological 
activity of interleukin-5 (IL-5). As a consequence, IL-5 antagonists have become an obvious target for therapy in this phenotype.

In this review, we will report the latest evidence on mepolizumab, a new and promising biological agent that binds to and inactivates IL-5, reducing the incidence of asthma exacerbations, even in patients with severe disease already on oral corticosteroids (OCS). ${ }^{9} 10$ The US Food and Drug Administration (FDA) has recently approved mepolizumab specifically for the treatment of severe eosinophilic refractory asthma.

\section{Eosinophils, IL-5, and asthma}

Eosinophils represent $1 \%-6 \%$ of the circulating white blood cells. They are important for protection against parasitic infestations and as mediators of allergic inflammatory responses. Different chronic disorders of the airways arise as a result of an inflammatory pathogenesis, in which eosinophils play a significant role and influence the degree of severity. ${ }^{11,12}$ In bronchial asthma tissue, eosinophilia is present in $40 \%-60 \%$ of cases; in the eosinophilic phenotype, blood and sputum eosinophils correlate with the severity of disease. ${ }^{13,14}$ The total amount of airway eosinophils in asthma can be reduced and controlled in most patients with the help of regular inhaled corticosteroids. ${ }^{15}$

However, even high doses of inhaled corticosteroids do not succeed in modifying persistent airway eosinophilia and reduce exacerbation incidence in about $50 \%$ of patients with severe asthma, who represent $5 \%-10 \%$ of all asthmatics. ${ }^{8,16,17}$

Eosinophils have IgE receptors and are able to internalize the antigen-antibody complexes, release inflammatory mediators (like major basic protein), and kill microbial agents. Their granules also contain mediators typical of allergic reactions, such as histaminase and arylsulfatase. A second important activity of eosinophils is the secretion of leukotrienes, which play a role in the pathophysiology of asthma by inducing bronchoconstriction and mucus hypersecretion (Figure 1). ${ }^{18}$

A rich scientific literature supports eosinophils to be pleiotropic multifunctional leukocytes involved in the "innate" immune response against foreign agents in the airways and in the modulation of the "adaptive" immunity cascade, maintaining local immunity and inflammation. At the same time, the release of granule proteins, free oxygen radicals, and cysteinyl leukotrienes can cause tissue damage.

The full life cycle of eosinophils from production in the bone marrow to reaching the site of inflammation, from

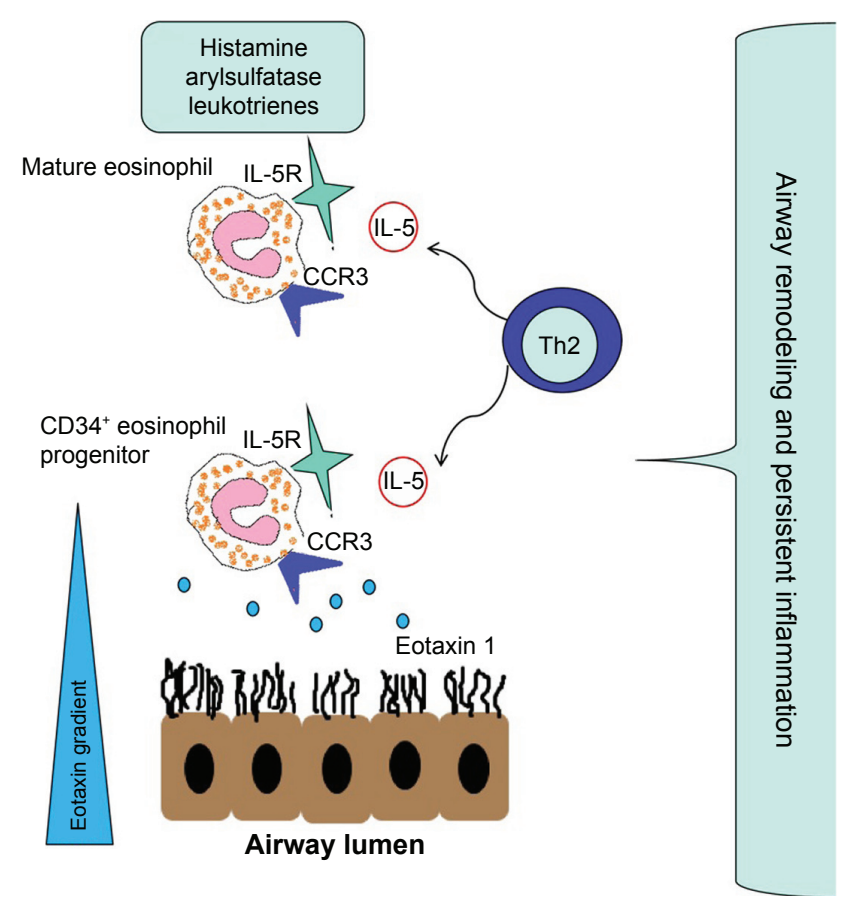

Figure I IL-5 and eotaxin role in recruitment, survival, and function of eosinophils. Abbreviation: IL-5, interleukin-5.

production to recruitment, from activation to apoptosis, is strictly modulated by IL-5 and its specific receptor (IL-5R) on target cells. ${ }^{12}$ There is evidence that differentiation of eosinophils occurs mainly in bone marrow but also in tissues undergoing an allergic response, such as in the bronchial mucosa of atopic asthma. ${ }^{19-21}$

The molecular structure of IL-5R is composed of a unique $\alpha$-chain (IL-5R $\alpha / \mathrm{CD} 125$ ) and the common cytokine $\beta$-chain ( $\beta \mathrm{c} / \mathrm{CD} 131)$. The IL-5R $\alpha$ binds specifically only to IL-5, while the $\beta$-chain can also bind IL-3 and granulocytemacrophage colony-stimulating factor through an extracellular domain.

Given these premises, an approach to anti-IL-5 therapy would seem to add significant value to usual therapy and help obtain a positive clinical response in eosinophilic asthma. Eotaxin 1/CCL11 is a related chemokine that can stimulate migration of eosinophil $\mathrm{CD} 34^{+}$progenitor cells in a dosedependent manner. The interaction between eotaxin 1 and its receptor CCR3 seems to be involved in the survival and activity of eosinophils in the airways independently of IL-5, influencing clinical symptoms and probably acting as an important driver of tissue events in asthma. The involvement of different cytokines in asthma may explain, at least in part, the discrepancy between eosinophil targeting and clinical response. One study demonstrated a decrease in blood and sputum eosinophils but no effect on late asthmatic response 
or airway hyperresponsiveness after administration of an anti-IL-5 antibody, thereby questioning the central role of eosinophils. Although several studies in vitro or on animal models and also in clinical trials have confirmed IL-5 inhibition as a potential effective approach for the treatment of severe asthma, clinical pharmacology has to take into account the emerging evidence on the interaction between eotaxin 1 and its receptor in developing new therapeutic approaches targeting the IL-5 pathway. ${ }^{22-26}$

\section{Mepolizumab features}

Mepolizumab is a humanized monoclonal antibody (mAb) antagonizing IL-5. Through a selective inhibition of eosinophilic inflammation, ${ }^{27,28}$ the agent reduces the number of eosinophils in sputum and blood, with important clinical outcomes such as a reduction of asthma exacerbations and a need for systemic glucocorticoids. ${ }^{10,25,29}$

Mepolizumab (SB-240563; GlaxoSmithKline, Research Triangle Park, NC, USA) was the first biological anti-IL-5 drug tested in randomized clinical trials (RCTs) on eosinophilic asthma and other eosinophilic diseases.

From a molecular point of view, mepolizumab is a humanized $\mathrm{mAb} \operatorname{IgG} 1 \kappa$, which is $N$-glycosylated, and it is formed by two light chains and two heavy chains bound by a disulfide bridge, with a global molecular weight of $149 \mathrm{kDa}$ (including $3 \mathrm{kDa}$ carbohydrate residues). ${ }^{30}$

After the initial studies on asthma, mepolizumab was licensed with the name Bosatria ${ }^{\circledR}$ for compassionate use in patients with eosinophil-related disorders - and in particular, the hypereosinophilic syndrome. The primary end point of lowering the prednisone dose $(\leq 10 \mathrm{mg} / \mathrm{d})$ was achieved in $84 \%$ of the patients treated with mepolizumab compared to $43 \%$ in those receiving placebo $(P<0.001)$. In the treatment group, the eosinophil count was normalized in $95 \%$ of subjects versus $45 \%$ of patients on placebo $(P<0.001) .{ }^{31}$

Finally, in November 2015, the FDA committee approved mepolizumab with the brand name Nucala ${ }^{\circledR}$ (GlaxoSmithKline) for use in patients older than 18 years with severe eosinophilic asthma at the dose of $100 \mathrm{mg}$ to be administered subcutaneously once every 4 weeks. ${ }^{32}$ In December 2015, the European Medicines Agency approved a marketing authorization valid throughout the European Union as "medicine under additional monitoring". ${ }^{33}$

\section{Mepolizumab in clinical practice: importance of asthma phenotypes}

At the beginning of experimental development, the most suitable asthma phenotype for treatment with mepolizumab was not clear. The results of the first studies were unsatisfactory, probably because of improper identification of potential responders, leading to doubts about the real effectiveness of this new molecule. Leckie et $\mathrm{a}^{25}$ were the first research group to test mepolizumab in an RCT on human subjects (Table 1). Unfortunately, the authors did not observe significant improvements in terms of airway hyperreactivity, peak expiratory flow, and forced expiratory volume in 1 second $\left(\mathrm{FEV}_{1}\right)$ in spite of a decreased airway and blood eosinophilia after 4 and 16 weeks. ${ }^{25}$

In a subsequent RCT, 362 patients with uncontrolled asthma despite inhaled corticosteroid therapy were evaluated to assess the clinical efficacy of intravenous (IV) administration at the dose of 250 or $750 \mathrm{mg}$ every 30 days. The administration of anti-IL5 mAb was related to a dramatic reduction of eosinophil levels in blood and sputum in both treatment groups. Unfortunately, clinical end points were not achieved; in particular, there was no significant decrease in exacerbation rates. One reason was probably that the authors did not consider the baseline level of airways eosinophilic inflammation as an important criterion. ${ }^{27}$

Actually, far more interesting results were achieved administering anti-IL-5 agents in patient groups with eosinophilic-related diseases. Nair et $\mathrm{al}^{29}$ assessed mepolizumab in a group of patients with significant sputum eosinophilia and uncontrolled asthma despite high-dose inhaled corticosteroids and OCS as standard treatment. The primary end point was the evaluation of the steroid-sparing effect of mepolizumab. In this regard in the mepolizumab group, a significant decrease $(P=0.04)$ was detected in the dose of prednisone, with an average reduction from 11.9 to $3.9 \mathrm{mg}$ in the treatment arm and a reduction from 10.7 to $6.4 \mathrm{mg}$ in the placebo group. There was also a significant reduction of eosinophils in blood and sputum, related to improvements in asthma control, with particular regard to scores on the Juniper Asthma Control Questionnaire (ACQ) and modest increase in $\mathrm{FEV}_{1}$ that were maintained for a period of 8 weeks. Moreover, in patients receiving mepolizumab, the median time to exacerbation was 20 weeks as compared to 12 weeks in the placebo group $(P=0.003)$. The authors showed a prednisonesparing effect in the mepolizumab treatment group, but there were no significant differences of OCS doses between the two subpopulations on study.

These data have underlined the need to identify reliable biomarkers able to predict the response to this therapy. ${ }^{29}$

A new study performed by Haldar et $\mathrm{al}^{9}$ on subjects with eosinophilic asthma (sputum eosinophilia $>3 \%$ ) and a history of severe recurrent exacerbations brought new data to 
support these considerations. In this study, the primary end point was the rate of severe exacerbation during treatment. Secondary outcome measures were improvement in asthma symptoms, Asthma Quality of Life Questionnaire score, postbronchodilator $\mathrm{FEV}_{1}$, airway hyperresponsiveness, and number of blood and sputum eosinophils. As expected, there was a significant reduction of eosinophils in blood and sputum. Over a 50-week period, patients enrolled in the mepolizumab treatment group had significantly fewer asthma exacerbations compared with the placebo group. ${ }^{9}$

A big step toward the understanding of the real therapeutic potential of mepolizumab was the "Dose Ranging Efficacy And safety with Mepolizumab" (DREAM) trial performed by Pavord et al, ${ }^{10}$ a multicenter, double-blind placebo-controlled study on patients with severe asthma presenting with one of the following features: either a sputum eosinophil count $>3 \%$, a fractional exhaled nitric oxide (FeNO) concentration $\geq 50 \mathrm{ppb}$, peripheral blood eosinophil count $\geq 300$ cells $/ \mu \mathrm{L}$, or rapid deterioration of asthma control after a reduction of $25 \%$ or less of regular inhaled or oral steroids. ${ }^{10}$ Treatment was carried out with one of three different doses of IV mepolizumab, 75, 250, and $750 \mathrm{mg}$, or matched placebo $(100 \mathrm{~mL} 0.9 \% \mathrm{NaCl})$. Among participants, 616 completed the trial with 13 infusions at 4 -week intervals. The primary outcome of the study was the rate of "clinically significant asthma exacerbations" in the 52 weeks of treatment and in the following 4 weeks. The definition adopted for clinically significant exacerbations was "worsening of asthma requiring the use of oral corticosteroids for 3 or more days, admission, or a visit to the emergency department". Also in this study, blood and sputum eosinophils were diminished in the active treatment group, but there were dose-related differences in the effect on sputum (but not blood) eosinophils. The lowest dose of mepolizumab, ie, $75 \mathrm{mg}$ IV every month, decreased exacerbations by $48 \%$ during the course of treatment. No additional significant gains in reducing asthma exacerbations were observed with 250 and $750 \mathrm{mg}$ of mepolizumab. The relation between mepolizumab and blood and sputum eosinophil counts indicates that the lowest dose has the best dose-response profile. No significant differences of $\mathrm{FeNO}$ values among the three subgroups were detected, confirming circulating eosinophils as the best biomarker of clinical response available at present. There are two other possible explanations. First, a number of exacerbations might not be eosinophil dependent, even if occurring in patients with high levels of eosinophils. Alternatively, mepolizumab is not able to fully control airway eosinophilia. It can be speculated that survival and activation of tissue 
eosinophils can be modulated by granulocyte-macrophage colony-stimulating factor or IL-3 as an alternative to IL-5dependent pathway. ${ }^{34,35}$ Effects on $\mathrm{FEV}_{1}$ and ACQ scores were only small and not statistically significant. The authors hypothesize that day-to-day symptoms may be distinct from exacerbations associated with severe asthma, and may "require different management strategies".

In the "Mepolizumab Treatment in Patients with Severe Eosinophilic Asthma" trial, Ortega et $\mathrm{al}^{36}$ randomized 576 patients with recurrent asthma exacerbations and eosinophilic inflammation despite high-dose inhaled corticosteroids, into one of three groups: $75 \mathrm{mg}$ IV mepolizumab, $100 \mathrm{mg}$ subcutaneous (SC) mepolizumab, or placebo. Exacerbation rates were reduced by both IV and SC mepolizumab (47\% and $53 \%$, respectively) as compared to placebo. Emergency department visits for a severe exacerbation were decreased by $32 \%$ in the IV group and $61 \%$ in the SC group. $\mathrm{FEV}_{1}$ and Asthma Quality of Life Questionnaire scores improved in both groups treated with IV and SC mepolizumab as compared to placebo. ${ }^{36}$ Blood eosinophil values were similar in the three groups at baseline, with an average of 295 cells/ $\mu \mathrm{L}$; eosinophil reduction started after 4 weeks from the administration, reaching the best result at Week 12. In a subgroup analysis, subjects with a blood eosinophil count $\geq 500$ cells $/ \mathrm{mm}^{3}$ showed better results than the other patients.

The Mepolizumab Treatment in Patients with Severe Eosinophilic Asthma study showed no difference in the clinical efficacy of $100 \mathrm{mg} \mathrm{SC}$ and $75 \mathrm{mg} \mathrm{IV}$, and the DREAM study showed no difference in the effects of 75,250 , and $750 \mathrm{mg}$ IV. ${ }^{10,36}$

According to these two studies, different dosing regimens and routes of administration produce similar clinical outcomes.

In the SIRIUS study by Bel et al, ${ }^{37} 135$ subjects with a history of at least 6 months of maintenance therapy with OCS (5-35 mg/d of prednisone or equivalent) were randomized to receive $100 \mathrm{mg}$ mepolizumab SC or placebo; the primary outcome was to assess the OCS-sparing effect of the active treatment. The mepolizumab group showed a decrease in the rate of exacerbations, and the relevant administration of systemic corticosteroids was reduced by $50 \%$, compared to no reduction in the placebo group. ${ }^{37}$

In a post hoc analysis of the DREAM study, a subpopulation of 188 OCS-dependent patients were enrolled. These subjects had received maintenance OCS (5-35 mg/d) for $\geq 6$ months and previous treatment with omalizumab without efficacy. In these subjects, mepolizumab showed a significant reduction in OCS use in the non-OCS and OCS groups and was able to reduce blood eosinophils and exacerbation rate during the 52-week treatment period. The reduction was higher in the OCS group. ${ }^{34}$

At present, a multicenter, double-blind, placebocontrolled parallel-group trial is underway, with the aim of evaluating the clinical effect of mepolizumab on healthrelated quality of life (HRQoL), other measures of asthma control, and main lung function parameters in a population with severe eosinophilic asthma. ${ }^{35}$

It is difficult to assess from the current literature to draw firm conclusions about the clinical role of mepolizumab in the real life of patients with asthma. Different studies provide demonstration that mepolizumab can improve HRQoL and reduce exacerbations in severe eosinophilic asthma.

A recent Cochrane systematic review ${ }^{38}$ evaluated whether mepolizumab treatment is better than placebo for patients with severe asthma. The authors compared eight studies that enrolled 1,707 patients with asthma. Six of these studies included only adults. Data analysis showed that patients with severe asthma and high levels of eosinophils had benefits from taking mepolizumab in terms of improved quality of life and reduced number of asthma exacerbations. Unfortunately, there was no improvement of lung function. In addition, according to the authors, some important aspects need further clarification and research, in particular, the dosage and length of treatment as well as the definition of which subgroups of patients can receive the best advantage from this treatment.

Another problem is that there are few studies on children and adolescents, so it is difficult to give indications in these subpopulations. An RCT regarding this is now recruiting 6-11-year-old patients with severe eosinophilic asthma with the aim to evaluate pharmacokinetics and pharmacodynamics of SC mepolizumab. ${ }^{39}$

\section{Toward tailored medicine}

Conventional asthma management is usually based on symptoms and lung function tests to evaluate airway obstruction and airway hyperresponsiveness. Following the development of new biological therapies, there is a growing interest in the identification of biomarkers useful in the diagnosis and management of asthma to be used in combination with clinical and functional data, which have no definite correlation with airways inflammation. Bronchoscopy with biopsies and bronchoalveolar lavage have been considered the gold standard until now (Table 2) to assess airway inflammation, but they have the disadvantage of being invasive and not always applicable in real-life settings. ${ }^{40,41}$ 
Table 2 Standard and new potential biomarkers useful for asthma diagnosis and management

\begin{tabular}{lll}
\hline Procedure/type of sample & Advantage & Disadvantage \\
\hline Bronchoscopy with biopsies and BAL & Present gold standard & Invasive procedure not very applicable in real-life setting \\
Eosinophils in induced sputum & Specific biomarker of airway inflammation & Sample not practical to collect \\
Peripheral eosinophilia & Easy blood sample collection & Subestimates value \\
Expired FeNO & Easily measurable with available device & Interpretation of results still controversial \\
Serum periostin & Easy blood sample collection & Data still controversial \\
\hline
\end{tabular}

Abbreviations: BAL, bronchoalveolar lavage; FeNO, fractional exhaled nitric oxide.

An ideal biomarker should be cheap, readily available, reproducible, minimally invasive, and clinically predictive, but unfortunately, none of the many molecules tested share all those characteristics.

The main parameters assessed so far in eosinophilic asthma were the level of eosinophils in sputum and blood. Induced sputum is not easy to obtain routinely, while circulating eosinophilia has only limited value in predicting airway pathology. In the DREAM study, blood eosinophil counts correlated with the response to mepolizumab, but the same did not happen with sputum eosinophilia. ${ }^{10} \mathrm{~A}$ post hoc analysis showed that a single peripheral blood eosinophil count $\geq 150$ cells $/ \mathrm{mL}$ at screening was a good predictor of response in patients with unstable asthma and numerous exacerbations. ${ }^{42}$ On the contrary, those subjects with baseline eosinophil count $<150$ cells $/ \mathrm{mL}$ had a limited reduction of asthma exacerbations. Since blood eosinophil levels can show spontaneous variations over time, a single measurement might not be sufficient to evaluate patients accurately, although this study has shown that a single analysis can be acceptable in RCTs. Another frequently used option is the expired FeNO, whose levels have a close correlation with airway eosinophils as confirmed in several studies. ${ }^{43}$ Data are still controversial, because according to some authors, $\mathrm{FeNO}^{44}$ correlates well with sputum eosinophils, whereas in another study, FeNO is correlated with sputum eosinophilia in only $78 \%$ of patients. ${ }^{45}$ These discrepancies have increased the uncertainties on the use of this biomarker in the management of IL-5 antagonizing agents. ${ }^{46}$

In recent years, an increasing interest has focused on serum periostin (or osteoblast-specific factor 2), a matrix protein secreted by bronchial epithelial cells after IL-13 stimulation. Periostin was originally identified in mesenchymal cells as osteoblasts, osteoblast-derived cells, and periosteum. Unfortunately, this serum protein does not correlate with sputum eosinophilia and eosinophilic airway inflammation. ${ }^{47}$ So far, it has been shown mainly to predict response to lebrikizumab (an anti-IL-13 mAb) ${ }^{46}$ Also in this case, current data are contradictory, because one study identified serum periostin levels as the best predictor of airway eosinophilia, ${ }^{48}$ whereas according to other authors, periostin and total IgE were not able to discriminate eosinophilic from non-eosinophilic asthma. ${ }^{45,46}$

At the moment, no ideal biomarker is applicable to the treatment with mepolizumab.

\section{Anti-IL-5 mAbs beyond mepolizumab}

In the last 15 years, anti-IL-5 mAbs other than mepolizumab were tested in clinical trials on asthma, in particular reslizumab and benralizumab.

Reslizumab is a humanized anti-IL-5 mAb (IgG4/א) binding circulating IL-5 with a high affinity, thus preventing the activation of its specific receptor. ${ }^{49}$ A preclinical study (Sch 55700) on animal models (allergic mice, monkeys, and rabbits) showed a long-term effect in reducing pulmonary eosinophilia and airway hyperresponsiveness..$^{50}$

Like mepolizumab, the first RCTs in patients with severe uncontrolled asthma on standard treatment were quite disappointing, in terms of $\mathrm{FEV}_{1}$ or symptom improvements, despite a significant reduction in circulating and sputum eosinophils. ${ }^{49}$ The clinical outcomes have shown an improving trend only once a specific hypereosinophilic asthmatic phenotype (sputum eosinophils $>3 \%$ and/or blood eosinophils 400 cells $/ \mu \mathrm{L}$ ) has been selected for treatment. In a Phase II trial on asthmatic patients with nasal polyposis, asthma symptoms improved significantly $(P=0.012)$ along with a slight amelioration in clinical control as measured by ACQ. ${ }^{51}$ In subsequent Phase III RCTs, significant improvements in $\mathrm{FEV}_{1}$ and ACQ score were found, ${ }^{52}$ particularly in the subgroup with nasal polyposis. ${ }^{53}$

Benralizumab (MEDI-563) is an IgG1 afucosylated antiIL-5Ra mAb that recognizes an epitope on IL-5R $\alpha$ close to the specific binding site for IL-5. In a preclinical study on primates, MEDI-563 induced antibody-dependent cell-mediated cytotoxicity of eosinophils and basophils, depleting blood eosinophils, and eosinophil precursors in the bone marrow. ${ }^{54}$ In Phase IIB RCTs on patients affected by severe asthma with blood hypereosinophilia, ie, eosinophil counts $>300$ cell $/ \mu \mathrm{L}$, 20 and $100 \mathrm{mg}$ SC benralizumab showed promising clinical 
results, in particular a significant reduction of exacerbation rate in comparison with placebo..$^{55,56}$

The effects of benralizumab on eosinophil counts and activity were evaluated in a very recent study on blood samples collected from asthma patients enrolled in two clinical Phase I and Phase IIa trials. A relevant anti-inflammatory effect was underscored through a significant reduction and a modulation of blood eosinophils, IL-5, eosinophil-derived neurotoxin, and eosinophil cationic protein. ${ }^{57}$

\section{Monitoring and duration of treatment}

The recent introduction of mepolizumab and the consequent lack of long-term studies does not allow to precisely define the appropriate follow-up. To our knowledge, there are only a few follow-up studies, not longer than 12 months. ${ }^{58}$

In our experience, evaluations useful for an effective and practical monitoring are represented by blood and sputum eosinophil counts, exacerbation rate, and score of HRQoL questionnaires, such as Asthma Control Questionnaire or Asthma Control Test. In clinical studies, no significant differences in $\mathrm{FEV}_{1}$ or FeNO have ever been seen. The ideal timing for clinical and laboratory monitoring could be initially after 4 weeks and then every 3 months, based on the pharmacokinetic and pharmacodynamic characteristics. ${ }^{58,34}$

At present, there are no other biomarkers useful for the follow-up of patients on mepolizumab treatment.

When to stop a therapy with mepolizumab or other biologics such as omalizumab is still a matter of debate. In a study on eight patients affected by hypereosinophilic syndrome and eosinophilic gastroenteritis, the withdrawal of anti-IL-5 treatment induced an increase of eosinophils to pretreatment levels, associated with a rebound of symptoms. ${ }^{59}$

A more recent prospective study evaluated the effects of the suspension of mepolizumab in a group of 27 subjects treated with the agent in the previous 12 months. ${ }^{58}$ A progressive increase of blood eosinophils to baseline counts over 6 months was found, along with a significant worsening in the rate of severe exacerbations after 12 months.

An RCT is in progress to evaluate whether patients with severe eosinophilic asthma who have received mepolizumab for at least 3 years need to continue this treatment to maintain clinical benefit. ${ }^{60}$

\section{Pharmacoeconomic aspects}

The significant progress in asthma therapy with the arrival of omalizumab and use of the new biological drugs and bronchial thermoplasty has increased the focus on the economic aspects because of the huge potential growth in direct costs related to those treatments.

Currently, there are still few data in literature due to the short time interval since the introduction of mepolizumab. Analysts and some early reports estimate a cost per year of treatment from $\$ 10,000$ to $\$ 15,000$ per patient. ${ }^{61}$ After FDA approval, the real price tag is $\$ 32,500$ per year per patient and approximately $\$ 2,700$ for a single 4 -week injection. To our knowledge, the only real cost-effectiveness analysis was recently conducted and published by the Institute for Clinical and Economic Review Group and was based on a simulation model of asthma outcomes and costs in a representative population of suitable patients to mepolizumab therapy. ${ }^{62}$ The authors evaluated the incremental cost-effectiveness of mepolizumab, applying drug costs obtained from current prices, and estimates of reductions in asthma exacerbations and OCS use from available clinical literature data. In a scenario analysis, the price of mepolizumab was determined that would produce cost-effectiveness results at willingness-to-pay thresholds of $\$ 50,000$ per quality-adjusted life year (QALY), $\$ 100,000$ per QALY, and \$150,000 per QALY, respectively. At the moment, based on current purchase prices, the costeffectiveness estimates are not affordable. To obtain a value correlated to the clinical benefit a discount of two-thirds to three-quarters from the current acquisition costs of mepolizumab would be necessary. According to the authors of this report, mepolizumab should have a value-based cost between $\$ 7,800$ and $\$ 12,000$ a year, whereas the full list price per patient in the USA is $\$ 32,500$ a year. Other doubts arise from the lack of clinical trials evaluating benefits in the long term.

Another group of researchers ${ }^{63}$ conducted a study with the aim to evaluate the cost-effectiveness of newest strategies for the treatment of severe refractory asthma, such as biologic drugs (omalizumab and mepolizumab) and bronchial thermoplasty. The authors used a theoretical model based on the US health care perspective, with a cohort of 10,000 adult patients affected by refractory asthma in an annual cycle and 10 -year time horizon. The addition of bronchial thermoplasty to biologic treatment in responder patients was found to be not cost-effective. However, in biologic nonresponders, bronchial thermoplasty remained a cost-effective option as an add-on treatment. Mepolizumab without bronchial thermoplasty was the most cost-effective option for biologic responders, with a 10-year per-patient cost of $\$ 116,776$ and 5.46 QALYs gained (Institute for Clinical and Economic Review: \$21,388). Bronchial thermoplasty is a cost-effective treatment option only in the nonresponders group to biologic treatment $(\$ 33,161$ per QALY). 
A recent draft guidance of the National Institute for Health and Care Excellence does not recommend mepolizumab as an add-on treatment for severe refractory eosinophilic asthma. This sharp conclusion is due to the fact that the costs of mepolizumab compared with usual asthma treatments are above the range usually considered to be a cost-effective use of National Health Service resources. ${ }^{61}$

In our opinion, as it occurred in the past for omalizumab, the increase in the number of eligible patients evaluated in clinical trials may dispel doubts about the real cost-effectiveness ratio of mepolizumab in clinical practice.

\section{Conclusion}

The prevalent opinion is to consider asthma as a syndrome composed of heterogeneous diseases; therefore, it has become clear that diagnosis and treatment may often need tailored approaches. Actually, if it is true that guideline-based therapies work in the majority of patients, there are still some with uncontrolled symptoms despite usual recommended therapies.

At present, the only treatment options available for severe uncontrolled asthma (Step 5 treatment of Global Initiative for Asthma guidelines) are omalizumab and OCS. OCS are often associated with several adverse effects, particularly when used as long-term therapy. ${ }^{64} \mathrm{~A}$ recent study found that $93 \%$ of subjects with severe asthma had one or more pathologic conditions related to systemic corticosteroids, such as cataract, obesity, type II diabetes, osteoporosis, dyspeptic disorders, hypertension, and so on, with a higher relative risk in corticosteroid-dependent asthma ${ }^{65}$ The search for new treatments must take into account as a priority the possibility to reduce the use of systemic steroids.

With new lines of study on mAbs against IL-5 and its receptor and on the recognition of specific biomarkers correlated with eosinophilia, mepolizumab has become a very interesting option for the treatment of these patients. The recent approval by the FDA advisory committee of the "real-life" usefulness of mepolizumab for patients with severe uncontrolled eosinophilic asthma is a step in this direction and will allow for the use of this therapy on a larger scale than now.

Among asthma phenotypes, the potential responders to mepolizumab treatment are patients with persistent systemic and airway eosinophilia $\left(>0.3 \times 10^{9} / \mathrm{L}\right.$ in blood, $>3 \%$ in sputum), possibly steroid responsive, with a poor symptom control, high dose of inhaled and systemic corticosteroids, heavy impact on quality of life, and several asthma exacerbations. A better clinical response is present in patients with eosinophilia $>500$ cells $/ \mu \mathrm{L} .{ }^{34}$ However, a multimodal approach taking clinical experience into account is necessary since the precise identification of potential responders needs further investigation. A correct selection of the eligible population would maximize the clinical benefits and reduce the need for systemic steroids.

There are many controversies about the optimal duration of treatment with anti-IL-5 mAbs. The conclusion of ongoing studies will probably allow a more precise answer to this and other important matters of debate, but at the moment, the available data would suggest to continue indefinitely.

Another major problem is the increase of direct costs for biologics in severe asthma, including mepolizumab, reslizumab, and benralizumab. Cost-effectiveness is a fundamental issue to better define the real-life usefulness of these drugs and to establish their correct position in treatment guidelines. Pharmacoeconomic studies carried out so far are controversial, but probably it will be necessary to act for a reduction of purchase costs to extend the availability of these promising therapeutic options.

\section{Disclosure}

Francesco Menzella and Luigi Zucchi participated in contracted research and clinical trials for Novartis, Sanofi, and GlaxoSmithKline. The other authors report no conflicts of interest in this work.

\section{References}

1. Custovic A, Johnston SL, Pavord I, et al. EAACI position statement on asthma exacerbations and severe asthma. Allergy. 2013;68:1520-1531.

2. Omachi TA, Iribarren C, Sarkar U, et al. Risk factors for death in adults with severe asthma. Ann Allergy Asthma Immunol. 2008;101:130-136.

3. Cisternas MG, Blanc PD, Yen IH, et al. A comprehensive study of the direct and indirect costs of adult asthma. J Allergy Clin Immunol. 2003;111:1212-1218.

4. European Network for Understanding Mechanisms of Severe Asthma. The ENFUMOSA cross-sectional European multicentre study of the clinical phenotype of chronic severe asthma. Eur Respir J. 2003;22: 470-477.

5. Heaney LG, Brightling CE, Menzies-Gow A, Stevenson M, Niven RM. Refractory asthma in the UK: cross-sectional findings from a UK multicentre registry. Thorax. 2010;65:787-794.

6. Moore WC, Bleecker ER, Curran-Everett D, et al. Characterization of the severe asthma phenotype by the National Heart, Lung, and Blood Institute's Severe Asthma Research Program. J Allergy Clin Immunol. 2007; 119(2):405-413.

7. Schäcke H, Döcke WD, Asadullah K. Mechanisms involved in the side effects of glucocorticoids. Pharmacol Ther. 2002;96:23-43.

8. Chung KF, Wenzel SE, Brozek JL, et al. International ERS/ATS guidelines on definition, evaluation and treatment of severe asthma. Eur Respir J. 2014;43:343-373.

9. Haldar P, Brightling CE, Hargadon B, et al. Mepolizumab and exacerbations of refractory eosinophilic asthma. N Engl J Med. 2009;360: 973-984.

10. Pavord ID, Korn S, Howarth P, et al. Mepolizumab for severe eosinophilic asthma (DREAM): a multicentre, double-blind, placebocontrolled trial. Lancet. 2012;380:651-659. 
11. Stone KD, Prussin C, Metcalfe DD. IgE, mast cells, basophils, and eosinophils. J Allergy Clin Immunol. 2010;125(2 Suppl 2):S73-S80.

12. Rothenberg ME, Hogan SP. The eosinophil. Annu Rev Immunol. 2006; 24:147-174.

13. Bousquet J, Chanez P, Lacoste JY, et al. Eosinophilic inflammation in asthma. N Engl J Med. 1990;323(15):1033-1039.

14. Wardlaw AJ, Brightling CE, Green R, Woltmann G, Bradding P, Pavord ID. New insights into the relationship between airway inflammation and asthma. Clin Sci (Lond). 2002;103(2):201-211.

15. Wenzel SE, Schwartz LB, Langmack EL, et al. Evidence that severe asthma can be divided pathologically into two inflammatory subtypes with distinct physiologic and clinical characteristics. Am J Respir Crit Care Med. 1999;160(3):1001-1008.

16. Woodruff PG, Modrek B, Choy DF, et al. T-helper type 2-driven inflammation defines major subphenotypes of asthma. Am J Respir Crit Care Med. 2009;180(5):388-395.

17. Wenzel S. Severe asthma in adults. Am J Respir Crit Care Med. 2005; 172(2):149-160

18. Becchetti E, Bani D, Baroni T. Istologia Umana. Napoli, Italy: IdelsonGnocchi; 2011:393-394. ISBN 9788879475419.

19. Rosenberg HF, Phipps S, Foster PS. Eosinophil trafficking in allergy and asthma. J Allergy Clin Immunol. 2007;119:1303-1310.

20. Molfino NA, Gossage D, Kolbeck R, Parker JM, Geba GP. Molecular and clinical rationale for therapeutic targeting of interleukin-5 and its receptor. Clin Exp Allergy. 2012;42:712-737.

21. Rossjohn J, McKinstry WJ, Woodcock JM, et al. Structure of the activation domain of the GM-CSF/IL-3/IL-5 receptor common beta-chain bound to an antagonist. Blood. 2000;95:2491-2498.

22. Sehmi R, Dorman S, Baatjes A, et al. Allergen-induced fluctuation in $\mathrm{CC}$ chemokine receptor 3 expression on bone marrow CD34+ cells from asthmatic subjects: significance for mobilization of haemopoietic progenitor cells in allergic inflammation. Immunology. 2003;109: 536-546.

23. Lamkhioued B, Abdelilah SG, Hamid Q, Mansour N, Delespesse G, Renzi PM. The CCR3 receptor is involved in eosinophil differentiation and is up-regulated by Th2 cytokines in CD34+ progenitor cells. J Immunol. 2003;170:537-547.

24. Park SW, Kim DJ, Chang HS, et al. Association of interleukin-5 and eotaxin with acute exacerbation of asthma. Int Arch Allergy Immunol. 2003;131:283-290.

25. Leckie MJ, ten Brinke A, Khan J, et al. Effects of an interleukin-5 blocking monoclonal antibody on eosinophils, airway hyper-responsiveness, and the late asthmatic response. Lancet. 2000;356:2144-2148.

26. Leckie MJ. Anti-interleukin-5 monoclonal antibodies: preclinical and clinical evidence in asthma models. Am J Respir Med. 2003;2: 245-259.

27. Flood-Page P, Swenson C, Faiferman I, et al. A study to evaluate safety and efficacy of mepolizumab in patients with moderate persistent asthma. Am J Respir Crit Care Med. 2007;176(11):1062-1071.

28. Menzies-Gow A, Flood-Page P, Sehmi R, et al. Anti-IL-5 (mepolizumab) therapy induces bone marrow eosinophil maturational arrest and decreases eosinophil progenitors in the bronchial mucosa of atopic asthmatics. J Allergy Clin Immunol. 2003;111:714-719.

29. Nair P, Pizzichini MM, Kjarsgaard M, et al. Mepolizumab for prednisone dependent asthma with sputum eosinophilia. $N$ Engl $J$ Med. 2009;360:985-993.

30. Zia-Amirhosseini P, Minthorn E, Benincosa LJ, et al. Pharmacokinetics and pharmacodynamics of SB-240563, a humanized monoclonal antibody directed to human interleukin-5, in monkeys. J Pharmacol Exp Ther. 1999;291(3):1060-1067.

31. Rothenberg ME, Klion AD, Roufosse FE, et al. Treatment of patients with the hypereosinophilic syndrome with mepolizumab. N Engl J Med. 2008;358(12):1215-1228.

32. U.S. Food and Drug Administration. FDA approves Nucala to treat severe asthma. FDA News Release. 2015 November 4. Available from http://www.fda.gov/NewsEvents/Newsroom/PressAnnouncements/ ucm471031.htm. Accessed January 10, 2016.
33. http://www.ema.europa.eu/ema. Nucala authorisation details [updated on 15/04/2016]. Available from: http://www.ema.europa.eu/ema/index. jsp?curl=pages/medicines/human/medicines/003860/human_med_. Accessed May 5, 2016.

34. Prazma CM, Wenzel S, Barnes N, Douglass JA, Hartley BF, Ortega H. Characterisation of an OCS-dependent severe asthma population treated with mepolizumab. Thorax. 2014;69(12):1141-1142.

35. GlaxoSmithKline. Efficacy and Safety of Mepolizumab as an Add-on Treatment in Chronic Obstructive Pulmonary Disease (COPD). Available from: https://clinicaltrials.gov/ct2/show/NCT02105961. NLM Identifier: NCT02105961. Accessed March 5, 2016.

36. Ortega HG, Liu MC, Pavord ID, et al. Mepolizumab treatment in patients with severe eosinophilic asthma. N Engl J Med.2014;371(13): 1198-1207.

37. Bel EH, Wenzel SE, Thompson PJ, et al. Oral glucocorticoid-sparing effect of mepolizumab in eosinophilic asthma. $N$ Engl J Med. 2014; 371(13):1189-2119

38. Powell C, Milan SJ, Dwan K, Bax L, Walters N. Mepolizumab versus placebo for asthma. Cochrane Database Syst Rev. 2015;7: CD010834.

39. GlaxoSmithKline. Pharmacokinetics and Pharmacodynamics of Mepolizumab Administered Subcutaneously in Children. Available from: https://clinicaltrials.gov/ct2/show/NCT02377427?term=mepolizuma b\&cond $=\% 22$ Asthma\%22\&rank=14. NLM Identifier: NCT02377427. Accessed May 26, 2016.

40. Lommatzsch SE, Martin RJ, Good JT Jr. Importance of fiberoptic bronchoscopy in identifying asthma phenotypes to direct personalized therapy. Curr Opin Pulm Med. 2013;19(1):42-48.

41. Djukanović R. Bronchoscopy as a research tool for the study of asthma pathogenesis and effects of antiasthma drugs. J Allergy Clin Immunol. 1996;98(5 Pt 2):S41-S45; discussion S64-S66.

42. Katz LE, Gleich GJ, Hartley BF, Yancey SW, Ortega HG. Blood eosinophil count is a useful biomarker to identify patients with severe eosinophilic asthma. Ann Am Thorac Soc. 2014;11(4):531-536.

43. Jatakanon A, Lim S, Kharitonov SA, Chung KF, Barnes PJ. Correlation between exhaled nitric oxide, sputum eosinophils, and methacholine responsiveness in patients with mild asthma. Thorax. 1998; 53(2):91-95.

44. Schleich FN, Seidel L, Sele J, et al. Exhaled nitric oxide thresholds associated with a sputum eosinophil count $\geq 3 \%$ in a cohort of unselected patients with asthma. Thorax. 2010;65(12): 1039-1044.

45. Wagener AH, de Nijs SB, Lutter R, et al. External validation of blood eosinophils, $\mathrm{FE}(\mathrm{NO})$ and serum periostin as surrogates for sputum eosinophils in asthma. Thorax. 2015;70(2):115-120.

46. Westerhof GA, Korevaar DA, Amelink M, et al. Biomarkers to identify sputum eosinophilia in different adult asthma phenotypes. Eur Respir $J$. 2015;46(3):688-696.

47. Izuhara K, Conway SJ, Moore BB, et al. Roles of periostin in respiratory disorders. Am J Respir Crit Care Med. Epub 2016 Jan 12.

48. Jia G, Erickson RW, Choy DF, et al. Periostin is a systemic biomarker of eosinophilic airway inflammation in asthmatic patients. J Allergy Clin Immunol. 2012;130(3):647-654.

49. Kips JC, O' Connor BJ, Langley SJ, et al. Effect of SCH55700, a humanized anti-human interleukin-5 antibody, in severe persistent asthma: a pilot study. Am J Respir Crit Care Med. 2003;167(12): 1655-1659.

50. Egan R, Athwal D, Bodmer M, et al. Effect of Sch 55700, a humanized monoclonal antibody to human interleukin-5, on eosinophilic responses and bronchial hyperreactivity. Arzneimittelforschung. 1999; 49:779-790.

51. Gevaert P, Lang-Loidolt D, Lackner A, et al. Nasal IL-5 levels determine the response to anti-IL-5 treatment in patients with nasal polyps. J Allergy Clin Immunol. 2006;118(5):1133-1141.

52. Bjermer CL, Maspero J, Ciesielska M, O’Brien C, Zangrilli J. A randomized phase 3 study of the efficacy and safety of reslizumab in subjects with asthma with elevated eosinophils. Eur Respir J. 2014; 44(58):299. 
53. Castro M, Mathur S, Hargreave F, et al. Reslizumab for poorly controlled, eosinophilic asthma: a randomized, placebo-controlled study. Am J Respir Crit Care Med. 2011;184(10):1125-1132.

54. Kolbeck R, Kozhich A, Koike M, Peng L, Andersson CK, Damschroder MM. MEDI-563, a humanized anti-IL-5 receptor alpha $\mathrm{mAb}$ with enhanced antibody-dependent cell-mediated cytotoxicity function. J Allergy Clin Immunol. 2010;125(6):1344-1353.

55. Ghazi A, Trikha A, Calhoun WJ. Benralizumab - a humanized mAb to IL-5Ralpha with enhanced antibody-dependent cell-mediated cytotoxicity - a novel approach for the treatment of asthma. Expert Opin Biol Ther. 2012;12(1):113-118.

56. Castro M, Wenzel SE, Bleecker ER, et al. Benralizumab, an antiinterleukin 5 receptor alpha monoclonal antibody, versus placebo for uncontrolled eosinophilic asthma: a phase $2 \mathrm{~b}$ randomised dose-ranging study. Lancet Respir Med. 2014;2(11):879-890.

57. Pham TH, Damera G, Newbold P, Ranade K. Reductions in eosinophil biomarkers by benralizumab in patients with asthma. Respir Med. 2016; 111:21-29.

58. Haldar P, Brightling CE, Singapuri A. Outcomes after cessation of mepolizumab therapy in severe eosinophilic asthma: a 12-month follow-up analysis. J Allergy Clin Immunol. 2014;133(3):921-923.

59. Kim YJ, Martin Prussin CB, Law MA, et al. Rebound eosinophilia after treatment of hypereosinophilic syndrome and eosinophilic gastroenteritis with monoclonal anti-IL-5 antibody SCH55700. J Allergy Clin Immunol. 2004;114:1449-1455.
60. GlaxoSmithKline. Cessation Versus Continuation of Long-Term Mepolizumab in Severe Eosinophilic Asthma Patients. Available from: https:/clinicaltrials.gov/ct2/show/NCT02555371. NLM Identifier: NCT02555371. Accessed May 4, 2016.

61. UK Medicines Information. New drugs online report for mepolizumab. Available from http://www.ukmi.nhs.uk/applications/ndo/record_view_ open.asp?newDrugID=4675. Accessed March 6, 2016.

62. ICER. ICER draft reports on Nucala ${ }^{\circledR}$ (mepolizumab) for asthma and Tresiba $^{\circledR}$ (insulin degludec) for diabetes posted for public comment edit. Boston, MA: ICER; 2015. Available from http://www.icer-review. org/icer-draft-reports-on-nucala-mepolizumab-for-asthma-and-tresibainsulin-degludec-for-diabetes-posted-for-public-comment. Accessed March 5, 2016.

63. Bogart M, Roberts A, Wheeler S. Cost-effectiveness of refractory asthma treatment strategies: a decision tree analysis. In: ISPOR 20th Annual International Meeting, Philadelphia, PA; 2015.

64. Global Initiative for Asthma (GINA). Global strategy for asthma management and prevention. GINA, 2015.

65. Sweeney J, Patterson CC, Menzies-Gow A, et al. Comorbidity in severe asthma requiring systemic corticosteroid therapy: cross-sectional data from the Optimum Patient Care Research Database and the British Thoracic Difficult Asthma Registry. British Thoracic Society Difficult Asthma Network. Thorax. 2016;71(4):339-346.
Therapeutics and Clinical Risk Management

\section{Publish your work in this journal}

Therapeutics and Clinical Risk Management is an international, peerreviewed journal of clinical therapeutics and risk management, focusing on concise rapid reporting of clinical studies in all therapeutic areas outcomes, safety, and programs for the effective, safe, and sustained use of medicines. This journal is indexed on PubMed Central, CAS,

\section{Dovepress}

EMBase, Scopus and the Elsevier Bibliographic databases. The manuscript management system is completely online and includes a very quick and fair peer-review system, which is all easy to use. Visit http://www.dovepress.com/testimonials.php to read real quotes from published authors. 